\title{
Load-out Design of Jacket With SPMT
}

\author{
Zheng Maoyao ${ }^{1, a}$, Wang Juan ${ }^{2, b}$ \\ ${ }^{1}$ Offshore Oil Engineering (Qingdao) Corporation Ltd, China \\ ${ }^{2}$ Offshore Oil Engineering (Qingdao) Corporation Ltd, China \\ azhengmy@mail.cooec.com.cn, bwangjuan@mail.cooec.com.cn
}

Keywords: SPMT, Axle load, Driving force, Stability, Friction force

Abstract. A new load out method with self-propelled modular transporter for jacket of offshore platform is introduced. The requirement of route selection, method axle force check, driving force check, stability check, friction force check and temporary structure design related to SPMT part during load out are discussed in detail in the article.

\section{Background Introduction}

QHD jacket is an 8-legs jacket of wellhead platform with 44 meters long, 20 meters wide and 30 meters height, and the weight is about 1600 tons. Refer to Fig. 1 for details. According to the traditional ways, it is should be fabricated on the skid way and then loaded out with dragging and slipping method. But there is no available skid way for fabrication of this jacket due to the recourse limit of construction yard. In order not to delay the delivery time, the jacket is arranged on the non-skid area to finish the construction. The change of fabrication yard condition lead to the jacket cannot be loaded out by dragging and slipping method, so a new load method with SPMT should by applied.

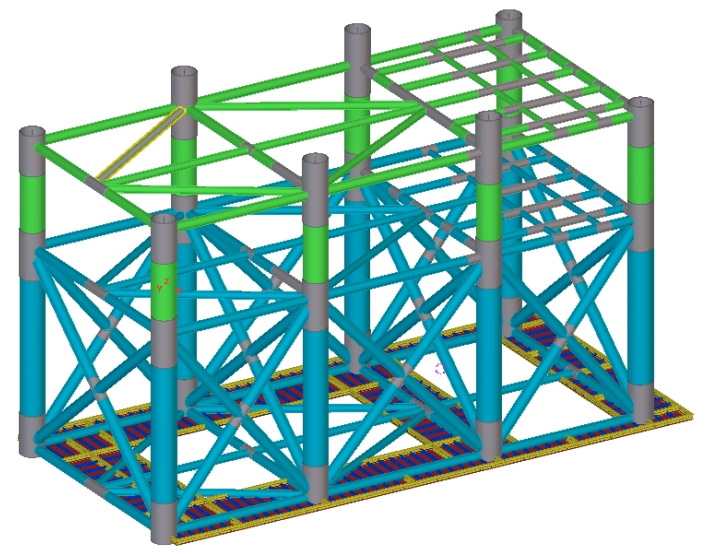

Fig. $13 \mathrm{D}$ view of QHD jacket

\section{General Introduction of SPMT}

SPMT, the abbreviation of Self-propelled Modular Transporter, is a new type of transportation facility which developed from the 1970s. [1] It is has been widely has been applying for heavy transport in various industries, such as civil, petrochemical etc. The SPMT is mainly consisting of two parts, car body bearing the cargo weight and power processing unit producing power to supply the driving force. The car body was categorized to various types of unit according to the wheel axles involved. 4, 6 or 8 axles unit are common types. The Fig. 2 shows the type of six axles unit of SPMT.

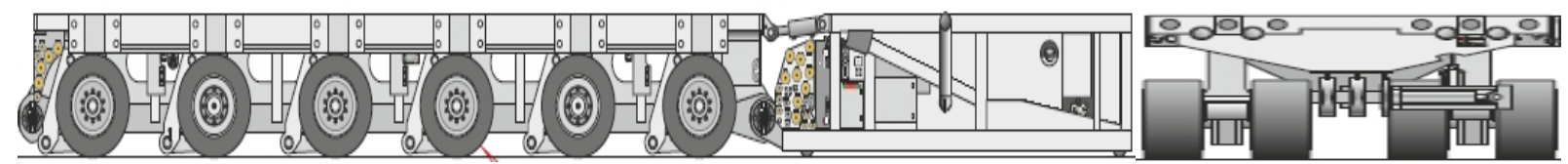

Fig. 2 six axles unit SPMT

Some advantages of SPMT makes it is selected and adopted to perform the load out of the jacket. It includes but not limited to the following items: 
1) Assemble conveniently. SPMT is designed modularly. Units could be quickly assembled through the high strength pin or bolts according to the cargo dimension and weight. Comparing to the dragging and slipping method, it saves more time in preparation phase.

2) Large load capacity. In theory, just from the view of SPMT itself, the cargo weight is not limited if the units quantity is enough. In 2009, a 15000 tons PQ module of GOYA project was loaded out by SPMT. It is the record at that time.

3) Low requirement of bearing capacity of ground. Carrying loads are equally distributed to each wheel axle with adjustment of hydraulic system. The max requirement is 10 ton per square meters with the rated load of 34 ton loaded on each axle.

4) Flexible turning. SPMT could be controlled to drive straight, diagonally, even rotation through the angle sensor. It gives more choice to the yard layout for construction.[2]

5) Adapt to complicated road conditions. The tire could be raised and declined through the suspension system . It makes SPMT could drive on the road with not so flatness surface and minor slope.

\section{Plan Design}

The content of load out design of jacket includes many contents. This chapter is concentrating on SPMT part. Axle load check, diving force check, stability check and friction force check are introduced in this chapter.

Transportation Route Selection. It is necessary to talk about the transportation route firstly. Although SPMT could cope with complicated road conditions, an optimal transportation route still has more positive effect, such as save more time cost, lower the safety risk. The following factor should be considered during the route selection.

1) The bearing capacity of ground shall be more than 10 tons square meters.

2) Minimize the times of turning.[3]

3) The slope of route shall be less than $2 \%$.

4) The ground should be flat as much as possible. No cavity which deep or height exceeds $300 \mathrm{~mm}$, the allowable adjustment range of suspension system, is existing.

5) Ground should not be too soft. Or else, wrinkling, bunker and rut are easily came out to resist the driving of the SPMT.

6) The big rubbles with sharp edge should be removed which may scuff the tire.

Axle Load Check. During transportation, all axles are divided into several groups through the connection of oil circuit. Each group support one point and the load will be distributed equally to all axles within the group. Usually, 3-point or 4-point support method will be selected. In this case, 3-point method is selected considering with the ground flatness of fabrication yard is not very good. A preliminary arrangement and group division plan is shown as Fig. 3.
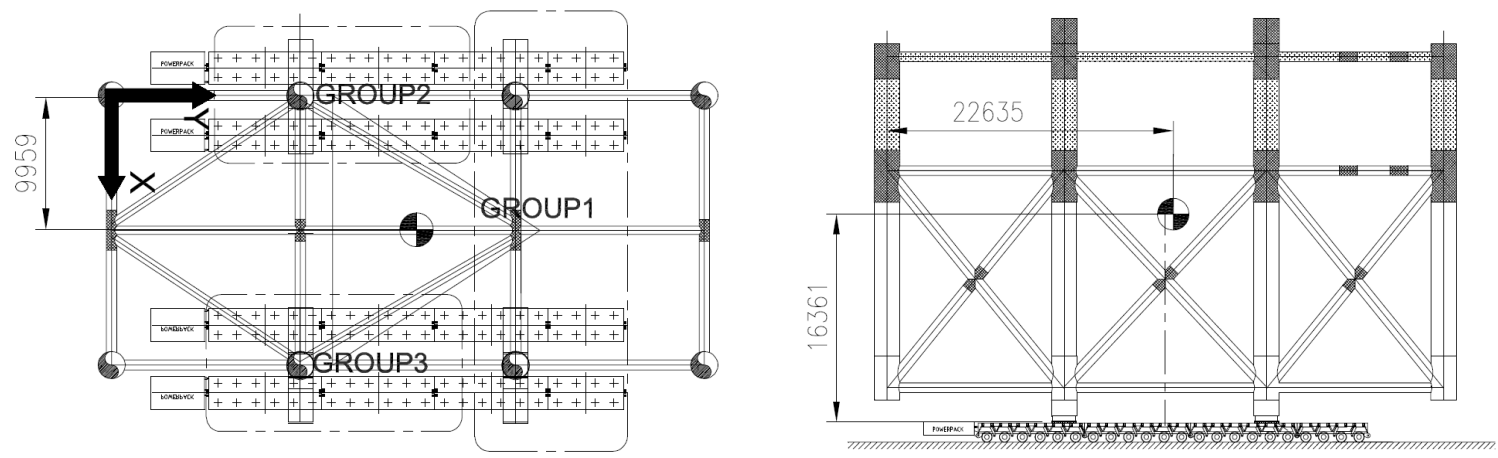

Fig. 3 Arrangement and group division plan

The axle load of each could be calculated with formula 1 . 
$\mathrm{f}_{\mathrm{i}}=\frac{\bar{F}_{\mathrm{i}}}{\mathrm{N}_{\mathrm{i}}} \quad \mathrm{i}=\mathbf{1}, 2,3$

$F_{1}$ : Distributed load on each group. By means of moment balance theory, we could get $F_{1=743.5[\text { ton], }} F_{2=648.7[\text { ton], }} F_{3}=641.8$ [ton]. It is noted the weight of carrier beam and SPMT itself shall also be considered when calculate' $F_{\text {: }}$.

$\mathrm{N}_{\mathrm{i}}$ : Quantity of axles of each group. As shown in Fig., $\mathrm{N}_{1}=32, \mathrm{~N}_{2}=28, \mathrm{~N}_{3}=28$.

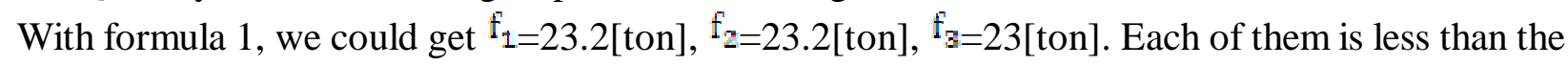
rated axle load of 34 [ton].

So it is ok.

Driving Force Check. The driving force should overcome the resistance caused by friction, Inertia, wind and slope of the route. The total driving force could be calculated with the for formula 2

$F_{d f}=f_{d} \times N_{d}$

$\mathrm{f}_{\mathrm{d}}$ : Maximum driving force produced by each driving, It is $60[\mathrm{KN}]$ refers to manufacture manual.

$\mathrm{N}_{\mathrm{d}}$ : Quantity of driving axles, 28.

After calculated with formula 2 , we get the $F_{\text {dE }}=1680[\mathrm{KN}]$

Besides of driving force, the adhesive force is also necessary and shall be checked with formula 3.

$F_{\mathrm{af}}=\mathbf{F}_{\mathrm{a}} \cdot \mu_{z} \cdot \cos \alpha$

$F_{\mathrm{d}}$ : Total axle load of all driving axles, 647.6[ton].

$\mu_{g}$ : Adhesion coefficient between tire and ground, it is 0.6 according to test record.

$\alpha$ : Road Slope, 1.15 degree;

After calculated with formula 3 , we get the $F_{\mathrm{af}}=3807[\mathrm{KN}]$

Meanwhile, we get the sum of resistance force is $1191.6[\mathrm{KN}]$. It is less than either driving force or adhesive force. So the driving force is enough, it is ok.

Stability Check. During the transportation, tilting moment produced by wind, ground slope, gyration, and transversal acceleration are tending to overturn the jacket. As shown in Fig. 4, the stabilizing angle should be calculated to check the stability of jacket with the formula $4 .{ }^{[4]}$

$$
\gamma_{\mathrm{i}}=\tan ^{-1}\left(\frac{\mathrm{L}_{\mathrm{i}}}{\mathrm{H} /}\right) \quad i=1,2,3
$$

$\mathrm{H}^{\prime}$ : Height of virtual gravity. The height should be measured from the axle.

$\mathrm{L}_{\mathrm{i}}$ : Stability arms. After calculation, $\mathrm{L} 1=5043 \mathrm{~mm}, \mathrm{~L} 2=5308 \mathrm{~mm}, \mathrm{~L} 3=5604 \mathrm{~mm}$.

According to the standard of GL Noble Denton, the stability angle should be calculated basing on the coordinate of virtual gravity caused by live load, such as wind etc. And when stability angle is greater than 8 degree, the transportation is safe without risk of tilting in theory. After calculated, $\alpha=16.13, \beta=16.93, \gamma=17.82$

The stability is OK. 


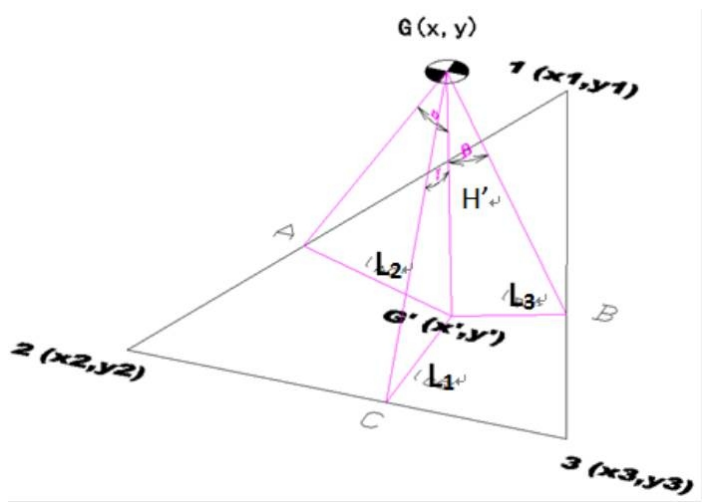

Fig. 4 sketch of stability

Friction Force Check. Friction force check is to check whether cargo will move when SPMT start and brake. It is also the standard to justify whether jacket is required to be lashed or strapped. The formula is shown as below.

$$
\mathrm{f}=\mathrm{m}_{\mathrm{o}} \cdot \mathrm{g} \cdot \cos \alpha \cdot \mu_{\mathrm{a}}
$$

$\mathrm{m}_{c}$ : Cargo weight, including jacket and carrier beam. It is 1682 [ton]

$\mu_{\mathrm{a}}$ : Static friction coefficient, 0.25 between steel and plywood

$\alpha$ : Ground slope, 1.15 degree

Meanwhile, we could calculate the total live load which makes jacket move on the SPMT though related formula. The result is shown in the table 1.

Table 1 Live load impact on cargo

\begin{tabular}{|c|l|l|l|l|l|}
\hline Direction & Inertia & Wind & Slope & Total & Unit \\
\hline X axis & 841 & 130 & 400 & 1374 & KN \\
\hline Y axis & 2523 & 59 & 400 & 2985 & KN \\
\hline
\end{tabular}

After calculated with formula 5, we get the friction force is $4944 \mathrm{KN}]$ which is greater than total live load in either $\mathrm{X}$ axis or $\mathrm{Y}$ axis direction. It means the jacket will not move when SPMT start or brake in theory. In order to increase the friction force, Rubber or plywood is often added between transportation support and SPMT. If the friction force is still not enough, lashing cargo on the SPMT with strap or turnbuckle shall be done.

\section{Temporary Structure Design}

Some temporary structures are essential to finish the load out job. It mainly include carrier beam and connection ramp shown in Fig. 5.

Carrier beam is designed to support jacket and spread the load on SPMT. The structure of carrier beam may different depending on the weight, dimension and profile of cargo.[5] Carrier beam designed for QHD jacket is shown in Fig. 6.It is consist of bottom frame, stiffen plate and piping stool.

Connection ramp is designed to connect wharf and barge for SPMT driving. It is often designed with three parts. Two of them fixed to the barge and wharf respectively. The last one is used to connect other two parts with hinge due to barge will move up and down along with the barge. 


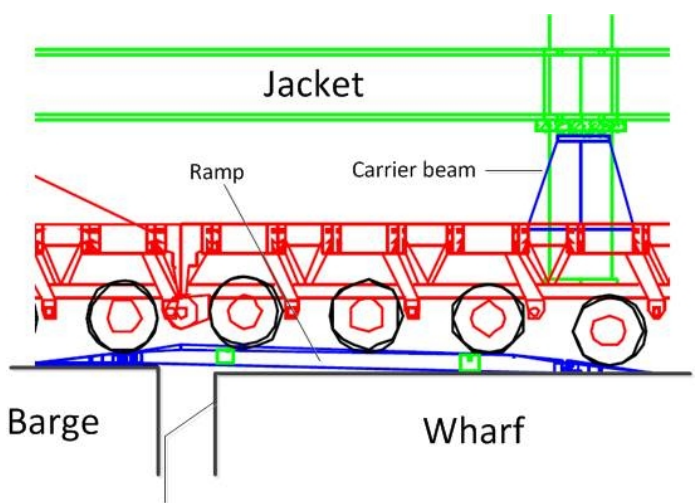

Fig. 5 Location of carrier beam and connection ramp

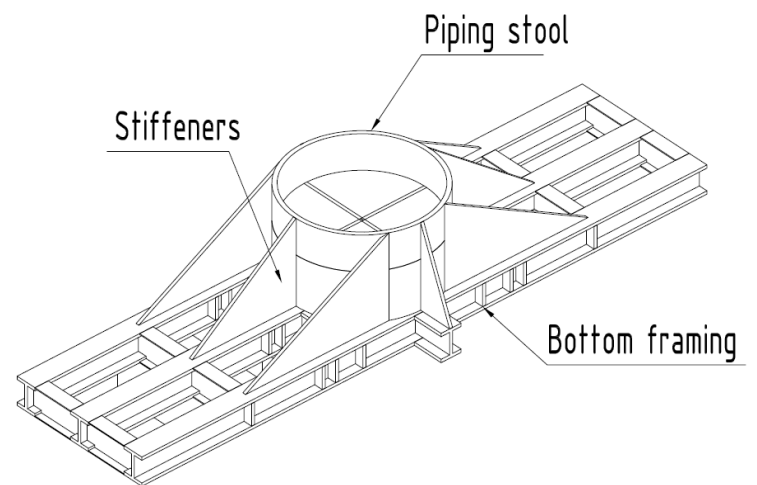

Fig. 6 Carrier beam details

\section{Steps of Load Out}

7) Combining units and checking basic functions of SPMT, such as braking, turning etc.

8) Driving SPMT to the appoint location under the jacket and connecting cable and oil circuit.

9) SPMT rising and stop when bear 50\% of total load, checking the distribution of load on each group. If no problem, continue raising SPMT till to take $100 \%$ of total load.

10) Lashing, if required.

11) Driving SPMT to the margin of ramp and make the final check of communication among each group.

12) Driving SPMT onto the barge. The gap between barge and ramp should be kept less than 15 centimeters via ballasting the barge.

13) SPMT declines and lay jacket onto the grillage, then sea fastening.

14) Driving SPMT off the barge.

\section{Conclusion}

The application of SPMT gives us more choice of load-out method for offshore structures. When this method is adopted, all contents mentioned in above chapters should be considered. Of course, it is only author own viewpoint, hope to be a reference for readers.

\section{References}

[1] Xiongbiao Wei, Kaifeng Wang etc.: Introduction of Load-out Method for Offshore Structure, Symposium of China Steel Structure Association Academic Annual Conference (2009) P.607-610.

[2] Xiaoke Wei, Xingmin Luo: Discussion of method for rolling the large-sized component onto deck barge, Journal of GUANGDONG Shipping Building, 4, 81-83, (2014).

[3] Liang Huang, Chuanhui Liu etc.: Discussion of optimization for loaf-out method of modular structure, Journal of Architectural Engineering Technology and Design, 3, 237, (2013).

[4] Hong Luo, Bing Li etc.: Load-out calculation of rolling method with SPMT, Journal of Petro \& Chemical Equipment, 16, 62-65. (2013).

[5] Sheng Yang, Zikun Zhao etc.: Ship-loading Technology and Conveying Frame Pre-installation of Large-sized Structures, Journal of Port Engineering Technology, 52, 42-44, (2015). 